\title{
Performance Enhancement of Bit Error Rate with Increased Capacity using Modified SIC-MUD for Polar Code based OFDM-IDMA System for $5 \mathrm{G}$
}

\author{
Hemlata Marne, Prachi Mukherji
}

\begin{abstract}
In the era of mobile data revolution there is a mass-market of smart phones culminating into an extensive growth in mobile services and applications. However, bandwidth is limited and valuable resource available. In this paper hybrid combination of OFDM-IDMA system is proposed using polar code as forward error correction code and Gold code based interleaver. It presents a method to improvebit error rate with 10 users and normalizedcarrier frequency offsets of 0.1 to 0.5.The simulation is done in the presence of Rayleigh and AWGN channel with 64 QAM modulation technique. A modified successive interference cancellation multi-user detection algorithm is proposed to cater multiple access interference.It is observed that proposed system withnormalized CFO of 0.1 for10 users at required SNR of $15 \mathrm{~dB}$ enhances the bit error rate to $1.021 * 10-40 v e r$ available existing systems. Hence the capacity of the system and data rate increases by three times to support multimedia application within available bandwidth.
\end{abstract}

\section{Keywords : OFDM, IDMA, CFO, MUD}

\section{INTRODUCTION}

Multiple-access interference (MAI) and inter-symbol interference (ISI) are significant wellsprings of losses in wireless communication frameworks. However, high information rate is favored in numerous current applications. Further, as the symbol timedecreases by the expansion of information rate, the systems utilizing single-carrier modulation experience the ill effects of extreme ISI brought about by the dispersive fading of wireless channels, bringing about the need of progressively complex equalization. Orthogonal Frequency Division Multiplexing (OFDM) is a multi-carrier modulation method where information symbols modulate a parallel accumulation of normally separated sub-carriers. The sub-carriers have the least frequency separation essential to keep up orthogonality of their associating time-domain waveforms, however the spectra of the signals relating to various sub-carrier's overlap in frequency domain. The spectral overlap results in a waveform that utilizes the accessible bandwidth with

Revised Manuscript Received on July 25, 2019.

Hemlata Marne, Research Scholar, Sinhgad College of Engineering, Department of Electronics and Telecommunications, Pune, Maharashtra, India

Prachi Mukherji, Professor \& Head, Cummins Collegeof Engineering for Women, Department of Electronics and Telecommunications, Pune, Maharashtra, India. exceptionally high bandwidth efficiency. However, OFDM suffers from carrier frequency offset (CFO). This is because of the fact that the way the sub carriers are dispersed intently in frequency. Imperfect frequency synchronization sources a misfortune in sub carrier orthogonality, which seriously debases execution of OFDM.Further, addition of cyclic prefix causes overhead in the OFDM framework.

On the other hand, Interleave Division Multiple Access (IDMA) conspire is proposed for the fifth generation mobile communication system that mitigates the effects of MAI by appointing unique interleavers for each user. This bring about conveying a high Bit Error Rate (BER) execution for a less signal to noise ratio (SNR) but recepient complexity increments with the number of paths.An OFDM-IDMA scheme waspresentedas an alternatetobasic IDMA over multipath channels to cater the impact ofMAI and ISI for $5 \mathrm{G}$ wireless communication systems. In this scheme, ISI is preserved by the cyclic prefixing method in OFDM, and MAI by iterative detection with IDMA. It giveshigher spectral and power efficiency. In any case, OFDM-IDMA experiences high affectability to carrier frequency offsets (CFOs) and high Peak to Average Power Ratio (PAPR). This multiple access strategy is utilized in digital audio and video broadcasting.In association, 3rd Generation Partnership Project (3GPP) standardization group considers polar codes as error correcting codes in $5 \mathrm{G}$ system.

In this paper, at first the impact of CFOin evaluating the performance in terms of BER for polar code based OFDM-IDMA system with proposed modified successive interference cancellation (mSIC) receiver is considered. This multicarrier- Multi-access method for mobile communication system is assessed dependent on WiMAX standardIEEE 802.16 with 64 QAM over Rayleigh channel models. Here the impact of symbol duration offsetis not considered. The proposed work is important because of its fruitful contribution in improving the BER of OFDM-IDMA framework.This paper is sorted out into various sections. Section 2displaysinsights of literature review. Section 3analyzes the proposed algorithm.Section 4shows a createdtest engine model utilized for simulation of proposed work. Section 5 tends to the implementation details of OFDM-IDMA

system.Simulation results

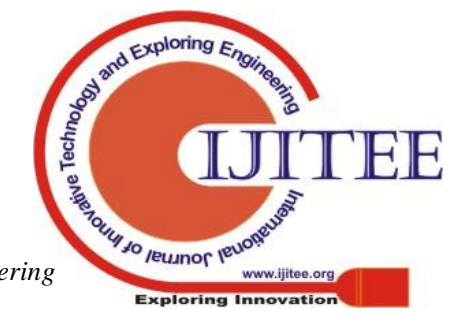


along with discussions and conclusionare exhibited in section 6 and 7 respectively.

\section{LITERATURE REVIEW}

Today mobile phone is the preferred medium for voice, data communication and multimedia applications. The reviewed literature is divided into two parts. The first part discusses the challenges that need to be considered for OFDM-IDMA system, The second one talk about the usefulness of polar codes as error correcting codes for performance enhancement of BER.

In [4] the demands of 5G wireless communication are listed. A multicarrier scheme like OFDM in view of Non-Orthogonal Multiple Access (NOMA) for 5G experiences high PAPR and affectability to CFO. These issues are settled utilizing proficient pre-coding procedures and an exceptionally simplified enhanced Minimum Mean Square Error (MMSE) recipient. The MMSE weights are planned in order to attain joint adjustment and CFO pay. In [5], a closed structure answer for the likelihood of error of MQAM- STBC-OFDM frameworks with frequency offsets and channel estimation errors in generalized frequency selective fading channels is inferred. Aside from this, the impact of imperfect CSI is likewise considered for error rate investigation.In [6]authors have announced that estimation blunders may augment by the amount of users in OFDM and IDMA conspire. To manage CFOs Space-Alternating Generalized Expectation-Maximization algorithm is proposed to separate the multiuser issue into various single-user issues. The proposed frameworks achieve better BER. In [7] authors have proposed versatile correction algorithm for OFDM IDMA frameworks in nearness of CFO in a fast fading multipath channel. A Least Mean Square (LMS) created versatile synchronization algorithm is applied to deal with CFO errors. Authors presumed that expansion in mobile speed debases the BER of the framework.Authors [8] have investigated and assessed the influence of $\mathrm{CFO}$ on the execution of OFDM IDMA scheme utilizing LMS based versatile rectification algorithm in a moderate just as quick fading multipath channel.Detection technique which alleviates the influence of CFOs in uplink OFDM-IDMA systems has presented in [9]. The iterative estimation discarded the joined obstruction from various users and CFOs at the recipient. The BER execution setback is a noteworthy issue in recipients due to impact of waiting CFOs.In [10] authors have presented exact signal model and novel CFO technique for OFDM framework which mitigates their destructive impacts. The proposed framework handles the $\mathrm{CFO}$ issue on the grounds that in it as per CFO estimation which depends on preambles for synchronization of current frame.Regularized correlated time averaged based variable forgetting factor recursive least square based channel estimation method for OFDM-IDMA systems has presented in [11]. This estimator has higher computational complexity.In [12] authors have discussed that pilot positions impact the execution of channel error estimation in OFDM-IDMA structures. In this manner for pilot arrangement process particle swarm improvement (PSO) figuring have proposed which diminishes the estimation errors in OFDM-IDMA structures.An epic inverse tree interleaver (ITI) in light of flip left-right methodology has been proposed for IDMA system in [13].This unique interleaver beats numerous other existing interleavers and requires less memory with no noteworthy trade off in the execution. A tale idea of user go parameter has been presented for deciding computation intricacy of ITI for every user. In [14] authors have examined the utilization of space time block coding (STBC) technique in OFDM IDMA scheme which offers extra dependability to wireless link. Utilization of STBC increase the complexity of OFDM IDMA structures since progressively number of transmit antennas are fundamental. In [15] joint detection and decoding (JDD) structure are proposed to decrease the decoding intricacy of polar-coded OFDM-IDMA conspire. It is given that a sign assisted JDD conspire further decreases the intricacy to half in contrast with that of JDD with no execution degradation at high SNR area. The polar-coded framework gives preferred BER execution over the un-coded scheme.

Authors [16] have proposed a multiple permuted factor graph-based decoder for polar codes. This belief propagation list (BPL) decoder is iterative in nature. Distinctive choice methodology of frozen bit positions can additionally improve the error rate execution of the suggested decoder yet it has high decoding multifaceted nature.Authors [17] have introduced that Successive cancellation (SC) deciphering has less computational multifaceted nature when contrasted and belief propagation (BP) disentangling, yet at the expense of long latency because of the sequential nature of the SC calculation. The proposed a novel plan depends on sub factor graph freezing which lessens the normal number of computations and iterations required by BP decoding. This system has low latency and low complexity.Author [18] has used the channel polarization effect to create codes that achieve the symmetric channel limit by polar coding. The exhibition of proposed polar codes with Belief propagation decoding algorithm is basically better than anything SC unwinding for more diminutive block length. In [19] various factors which impact the execution of multiuser Ultra Wide Band (UWB) channel based OFDM structure have inspected. The use of the polar code prescribed for better bit error performance. The multifaceted idea of the suggested polar-coded structure is considerably lower than the LDPC coded scheme.Authors [20]displayedcurrent advances in capacity approaching codes. The paper gives an outline of polar codes, spatially coupled codes, LDPC codes. Coding hypothesis and coding applications from various creators, spurs to proceed with research in the entrancing region of limit capacity approaching codes. In [21] an essential discrete inquiry heuristic algorithm has presented to find the best structured SNR for building up the polar code. It is seen that at high structure SNRs, the BER execution of the system spoils with a development in designed SNR for all the development algorithms.In [22] authors have exhibited the two innovative channel coding strategies, LDPC codes and polar codes embraced by the $5 \mathrm{G}$ new radio (NR) standard. It is demonstrated that the NR polar codes by and large beat the LTE turbo codes particularly at low code rates however at the expense of higher complexity. The 5th generation wireless systems 
(5G) standardization process of the $3 \mathrm{GPP}$ picked polar codes as a channel coding system.

Several algorithms are suggested in the literature to enhance the BER in the presence of CFO and increasing number of users for various channels. Further, researchers have also proposed a simplified version of Successive Cancellation decoding algorithm to reduce decoding complexity and latency. Still there is a challenge to develop multiuser multi carrier system to adopt higher modulation method in the presence of fading channels to achieve high data rate as demanded by $5 \mathrm{G}$ system at desirable BER for multimedia applications.

\section{PROPOSED MODIFIED SUCCESSIVE INTERFERENCE CANCELLATION MULTIUSER DETECTION ALGORITHM}

The main motivation behind the modified successive interference cancellation multiuser detection (mSIC-MUD) receiver is to minimize the effect of error propagations caused by incorrect detection of user symbols. This algorithm relies on the SIC of stronger user interferences for the benefit of weaker user signal detection.The subcarriers have different gain and SNR results in different symbol error rate. Therefore, from the knowledge of sub channel gain of the subcarriers and their receiver outputs, find the sub channels with lowest gains and lowest SNR. The complete performance of the scheme is enhanced by estimating the poor sub channels. The effect of error propagationcaused by detection of weakest signal first is minimized by adapting the channel behavior.ThemSIC-MUD algorithm is summarized as [3]

- Rank all M users in the order of descending signal strength, Pigi ${ }^{2}$.

Assumption is that $P_{1} g_{1}^{2}>P_{2} g_{2}^{2}>\ldots .>P_{M} g_{M}^{2}$

(1)

$$
\text { Let } y_{1}^{(i)}=r_{k}^{(i)} \text { and } l=1
$$

- Consider frequency response of the FIR channel given as

$$
H\left(e^{j 2 \pi f T}\right)=\sum_{k=0}^{L} h[k] e^{-j 2 \pi f k T}
$$

Where $L$ is channel order and $T$ is symbol duration. The pilot symbols help to gain knowledge of channel response in determining moderate, poor and good channels. Here only moderate and good channels are considered to enhance BER.

- Prepare a look up table to store data of channel gain and subcarrier index to estimate the count of moderate, poor and good channels. If the count of moderate and good channels is less or equal to count of poor channels, reject that frame. Otherwise go for detection of frames by detecting the signal of subcarrier having maximum channel gain.

- Detect the $l^{\text {th }}$ (strongest) user symbol by,

$$
\hat{s}_{k}^{(l)}=\operatorname{dec}\left(y_{l}^{(l)}\right)
$$

- Cancel the first strongest user interference from the received signals

$$
y_{l+1}^{(i)}=y_{l}^{(i)}-g_{l} \sqrt{P_{l}} R_{i, l}(k) \hat{\wedge}_{k}^{(l)} \quad i=l+1, \ldots \ldots, M
$$

(4)

- let, $l=l+1$ and repeat step 2 until $l=\mathrm{M}$

- Stop the detection process.

SIC-MUD receiver requires very little computation, since the interference cancellation step requires only. $O\left(M^{2}\right)$ complexity

\section{SIMULATION OF TEST ENGINE MODEL FOR OFDM-IDMA SYSTEM}

As the channel estimation execution is influenced by the places of pilot tones, the improvement of pilot arrangement process is known to be very significant on limiting the estimation errors, and it is appeared to be the noteworthy assignment for the multicarrier transmission advances. This paper contributes a new mSIC-MUD based receiver. Polar codes have been the subject of dynamic research as of late, chiefly because of the way that they are the primaryincessantly provably capacity accomplishing codes, with explicit construction and exceptionally low complex nature of encoding and decoding. The polar codes were developed by ErdalArikan [18] utilizing a novel idea called channel polarization. Before long, both the idea of channel polarization just as polar codes has been reached out to various applications and generalizations.

Figure 1 demonstrates the block schematic of the proposed OFDM-IDMA polar code based framework. At the transmitter side the binary input information is encoded by a polar encoder. The encoded information is interleaved and mapped onto 64-QAM values. Interleaving randomizes the occurrence of bit errors before interpreting. The IFFT modulates a block of input 64-QAM values onto 2048 subcarriers. A Rayleigh flat fading channel is used for signal propagation. 

OFDM-IDMA system for 5G

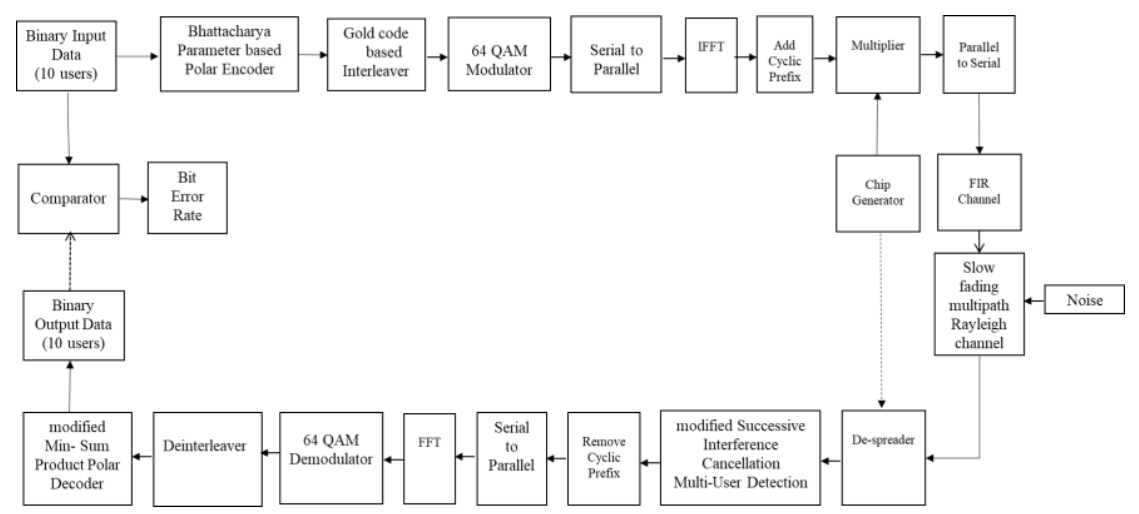

Fig.1: Block Schematic of Test Engine model of Polar coded OFDM-IDMA system

At the receiver section, the mSICworks on the detection of strongest user QAM symbols using matched filter receivers designed for single users. Therefore, once the strongest symbols are known at the receiver its interference effect gets cancelled on remaining user signals. Hence only one less than total number of user's unknown symbols remains for detection. Further the next strongest user signal is detected more accurately since the removal of strongest interference signal. Its effect this process continues till the weakest user signal is detected at the last. is subsequently gets cancelled from received signals that benefits two less than total number of users remaining symbols. The subcarriers are demodulated by an FFT which plays out the reverse activity of an FFT. The output of the FFT comprises QAM values that are mapped onto binary values. To effectively delineate the QAM values onto binary values, first the reference phases and amplitudes of all subcarriers are procured. Further, the reverse permutation is performed before polar decoding. Practically speaking despite the fact that most subcarriers are identified without errors, the general BER is overwhelmed by couple of subcarriers with the littlest amplitudes. A min-sum product polar decoding algorithm is used to produce binary output data with errors [23]. The binary input data is compared with binary output data to evaluate BER at different values of SNR, CFO and number of users. The logical BER expression for M-ary QAM signaling in AWGN and Rayleigh channels are given as [24]

$$
\begin{gathered}
P e=\frac{2(M-1)}{M \log _{2} M} Q\left(\sqrt{\frac{6 E b}{N_{0}} \cdot \frac{\log _{2} M}{M^{2}-1}}\right)(5) \\
P e=\frac{(M-1)}{M \log _{2} M}\left(1-\sqrt{\frac{3 \gamma \log _{2} M /\left(M^{2}-1\right)}{3 \gamma \log _{2} M /\left(M^{2}-1\right)+1}}\right.
\end{gathered}
$$

where $\gamma$ and $\mathrm{M}$ denote $\mathrm{Eb} / \mathrm{N} 0$ and the modulation order. Simulation of the OFDM-IDMA system has been done using polar code to show error correction proficiency in terms of reduced BER.Figure 2 shows comparative BERexhibition of turbo code based and polar code based OFDM-IDMA system using proposed Gold code based interleaver over fixedCFOof 0.1 for 10 users in presence of AWGN as well as Rayleigh channel.A one-half rate polar code is used to encode the input bits. The coded bits are interleaved with proposed interleaving mechanism that helps to separate adjacent bits by several bits as per the estimated values from the channel. The interleaved bits are then modulated onto 2048 OFDM subcarriers using 64-QAM. It can be seen from the curves that in the presence of AWGN channel using polar code at SNR equals to $15 \mathrm{~dB}$, results in a BER of 7.00512*10-5 whereas use of one-half rate turbo code achieves BER of only $1.00233 * 10-5$. In the presence of flat fading Rayleigh channel BER of $1.04602 * 10-4$ is obtained using polar code based system which is better than that of achieved byuse of turbo code based system. 


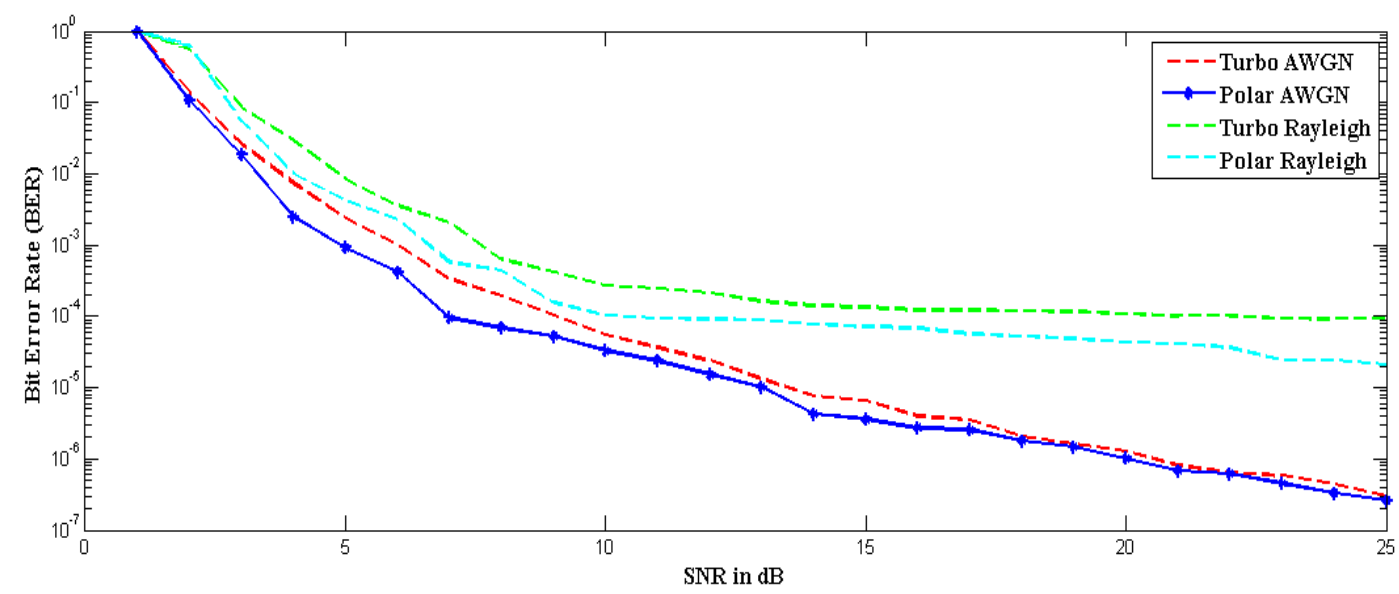

Fig.2:BER vs SNR performance comparison between proposed Turbo code based and Polar code based OFDM-IDMA system for 10 users over AWGN and Rayleigh channels where CFO is fixed to 0.1

Figure 3 shows that at fixed CFO of 0.5 and SNR of 5 $\mathrm{dB}$,increasing number of users from 1 to 10 degrades BER to $4.0823 * 10-2$ in flat fading scenario. It is observed that for lower number of users better BER is achieved thanin the presence of higher number of users. Figure 4displays simulated BER of polar code based OFDM-IDMA system for 10 users at $15 \mathrm{~dB}$ fixed SNR. It is clear from the curve that increase in CFO affects the BER in presence of AWGN and Rayleigh channels.

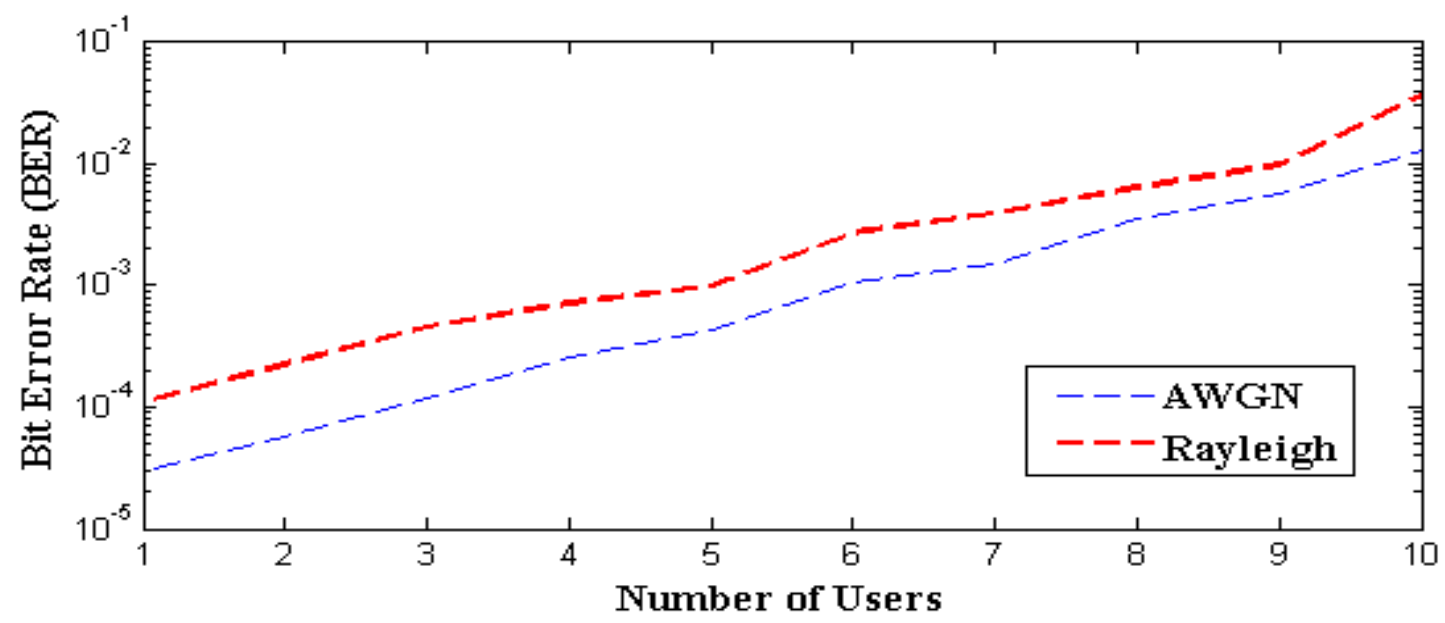

Fig. 3: BER vs number of user's performance of Polar code based OFDM-IDMA system over AWGN and Rayleighchannels for fixed CFO of 0.5 and SNR of $5 \mathrm{~dB}$ 
Performance Enhancement of Bit Error Rate with increased capacity using modified SIC-MUD for Polar code based OFDM-IDMA system for 5G

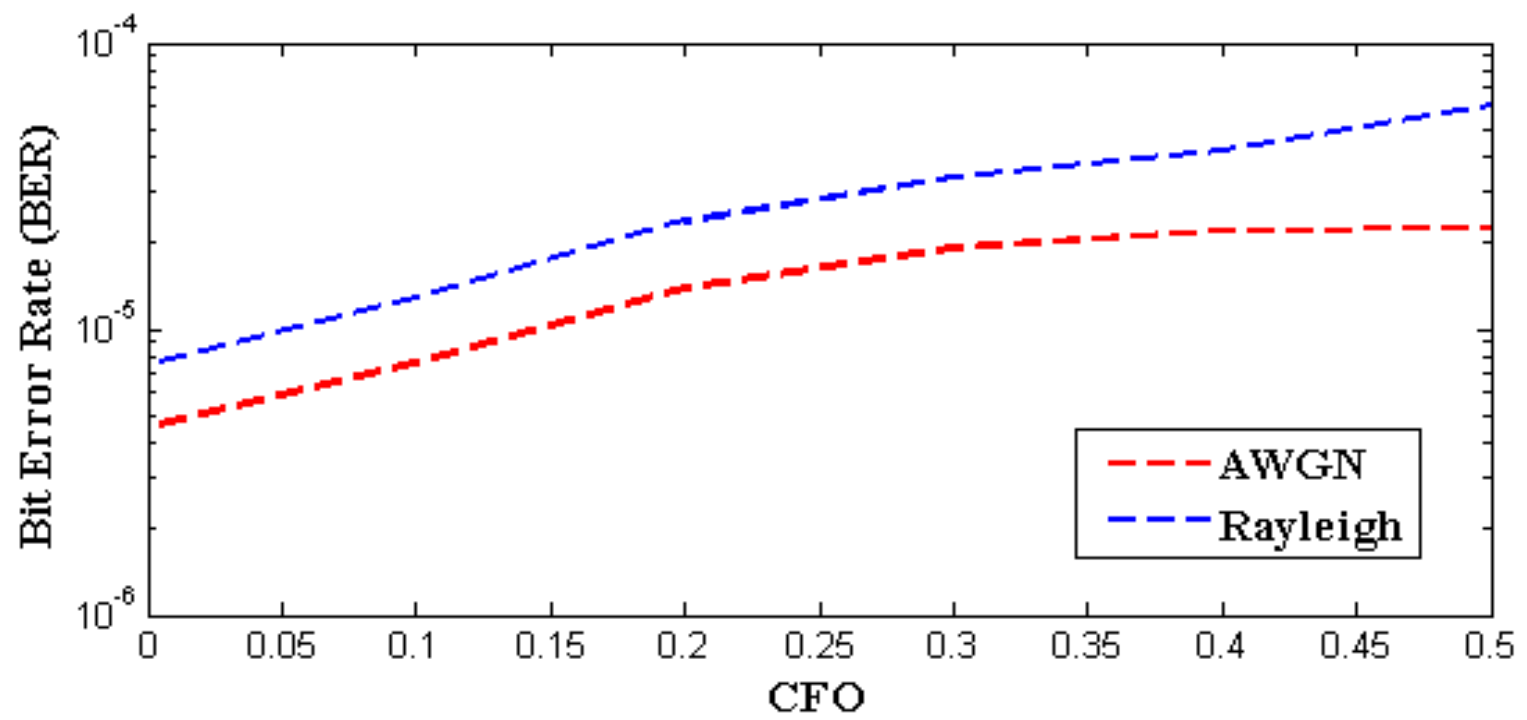

Fig. 4: BER vs CFO performance of Polar code based OFDM-IDMA system over AWGN and Rayleigh channels for 10 users at fixed SNR of $15 \mathrm{~dB}$

It is observed from theaforementioned curves that a tradeoff is possible between selection of number of users and acceptable CFO for achieving desired BER for minimum SNR.

\section{IMPLEMENTATIONOF OFDM-IDMA SYSTEM}

A python script is written to implement OFDM-IDMA system to evaluate the performance in terms of BER. The code is ported on raspberry pi board to transfer bit information from source to destination.

\begin{tabular}{|l|}
\hline Function : Assigning data to subcarriers \\
\hline INPUT : (k) \\
OUTPUT : (QAM_payload) \\
defOFDM_symbol(QAM_payload): \\
symbol = np.zeros(K, dtype=complex) \# the overall K \\
subcarriers \\
symbol[pilotCarriers] = pilotValue \# allocate the pilot \\
subcarriers \\
symbol[dataCarriers] = QAM_payload \# allocate the \\
pilot subcarriers \\
return symbol \\
OFDM_data = OFDM_symbol(QAM)
\end{tabular}

\begin{tabular}{|l|}
\hline Function : Rayleigh channel \\
\hline INPUT $:(\mathrm{g})$ \\
OUTPUT $:(\mathrm{r} 1)$ \\
\# Generating first Gaussian RV set \\
$\mathrm{g}=\operatorname{randn}(\operatorname{int}(\mathrm{O} / 2))+1 \mathrm{j} * \operatorname{randn}(\mathrm{int}(\mathrm{O} / 2))$ \\
$\mathrm{gc}=\mathrm{np} \cdot \mathrm{conj}(\mathrm{g})$ \\
$\mathrm{gcr}=\mathrm{gc}[::-1]$ \\
$\mathrm{g} 1=\mathrm{np} \cdot \mathrm{concatenate}((\mathrm{gcr}, \mathrm{g})$, axis $=0)$ \\
\# Generating second $\mathrm{Gaussian} \mathrm{RV} \operatorname{set}$ \\
$\mathrm{g}=\operatorname{randn}(\operatorname{int}(\mathrm{O} / 2))+1 \mathrm{j} * \operatorname{randn}(\mathrm{int}(\mathrm{O} / 2))$
\end{tabular}




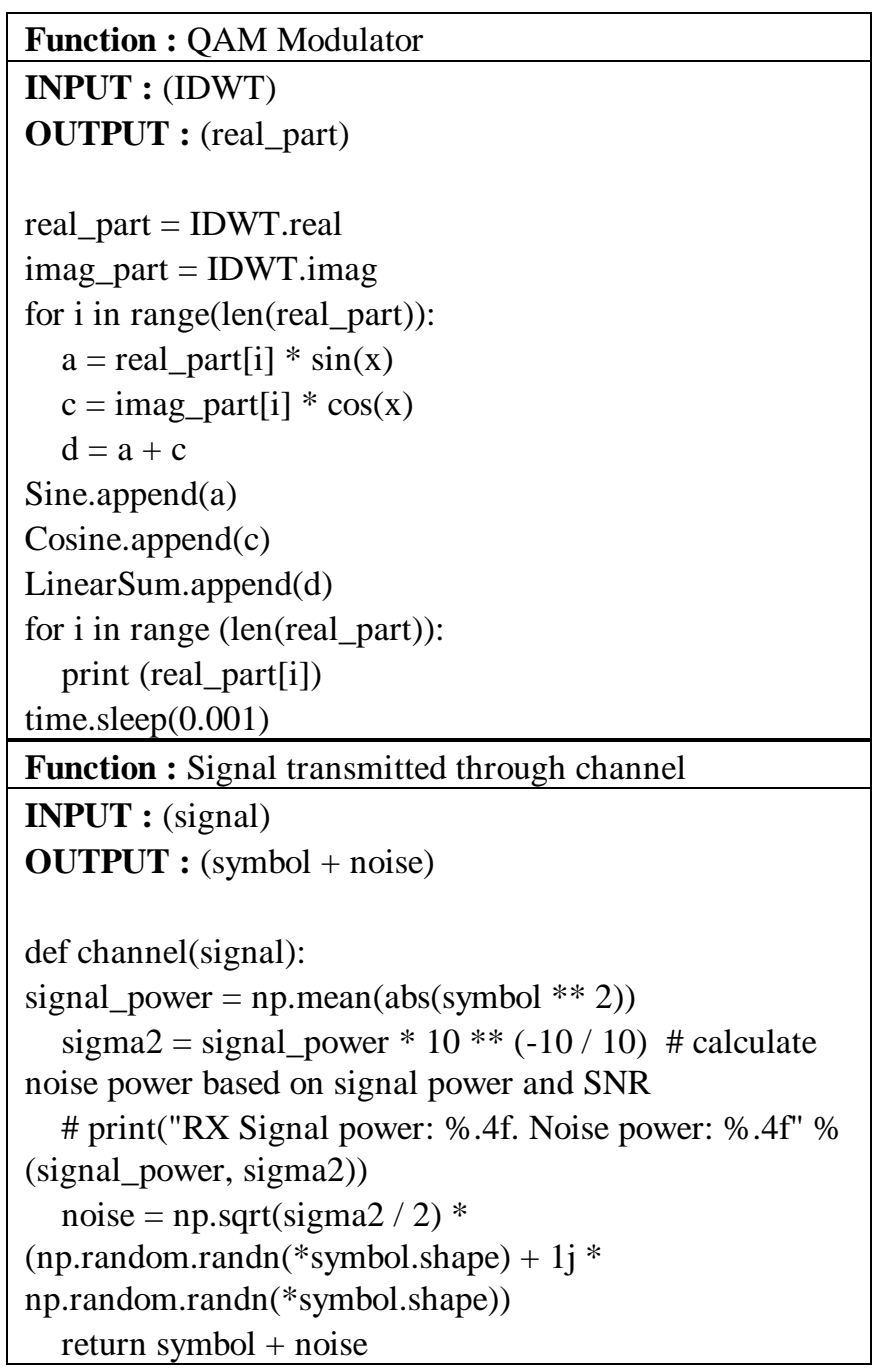

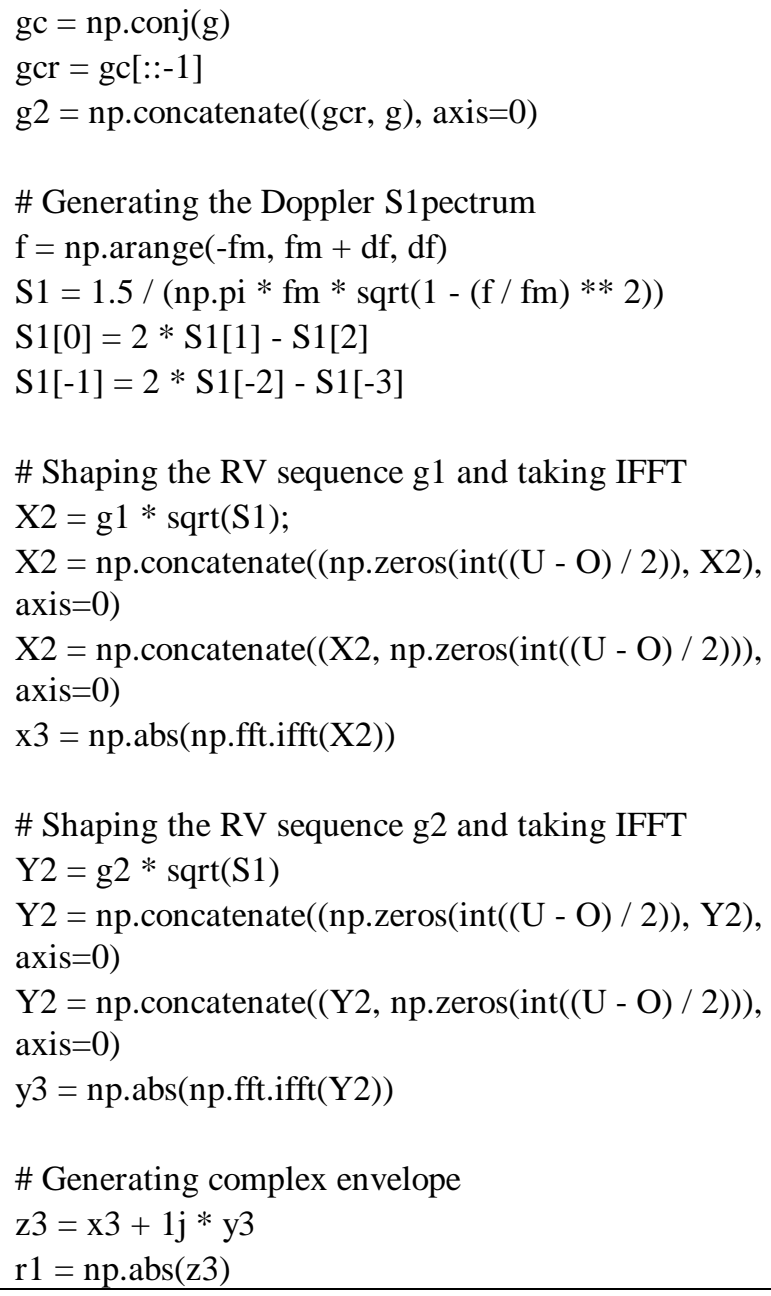




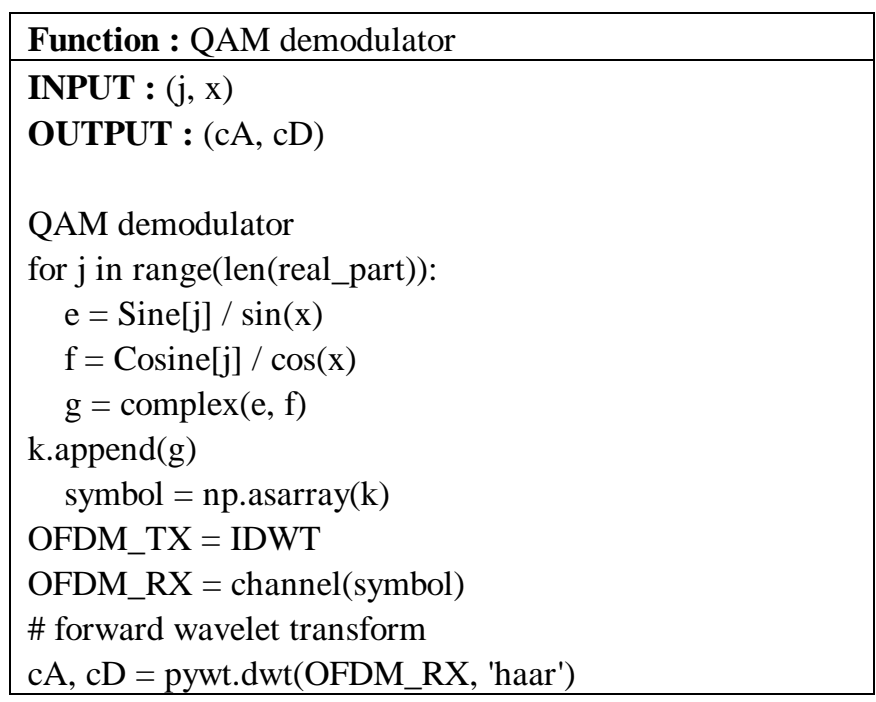

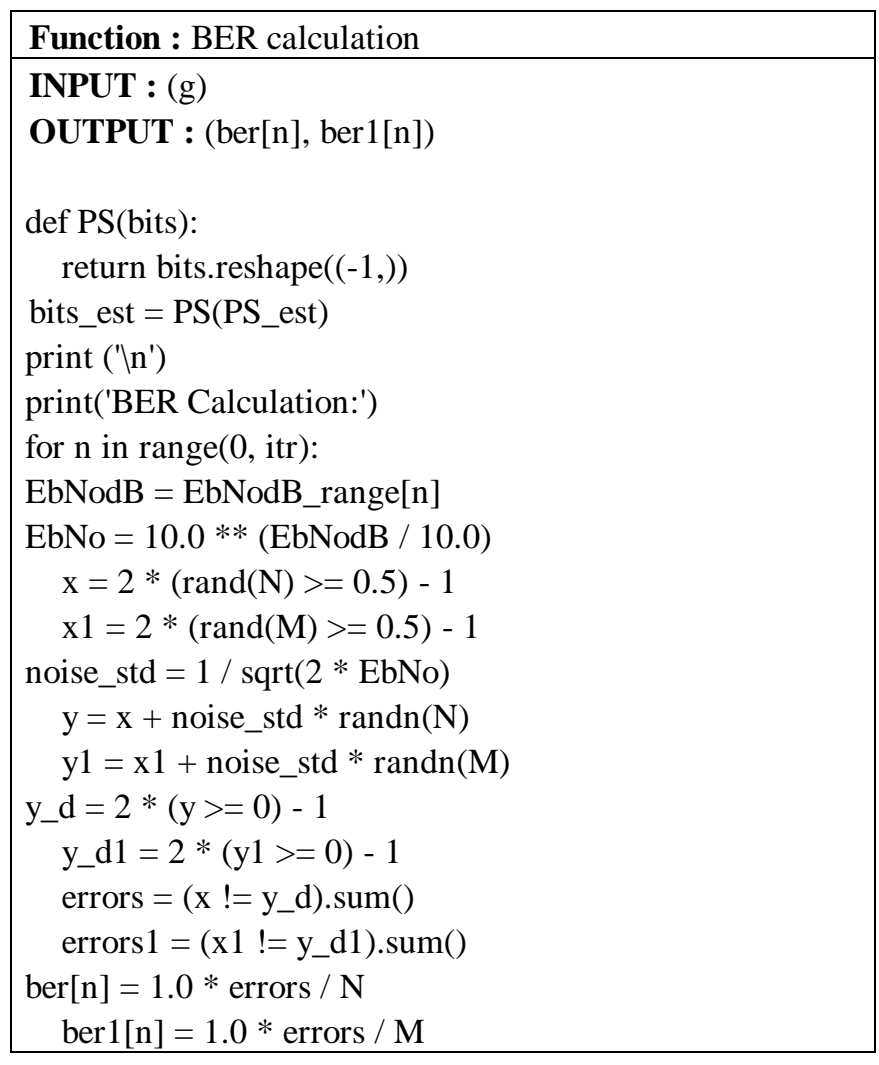

\section{RESULTS AND DISCUSSION}

OFDM-IDMA scheme was proposed with polar code as FEC alongside Gold code based interleaver to assess performance as far as BER in existence of CFO and number of users. The simulation was done within the sight of Rayleigh and AWGN channel. It is seen from table 1 that proposed polar coded OFDM-IDMA framework accomplishes desired BER for least SNR.

Table 1.Comparative analysis of Turbo coded system with proposed Polar coded OFDM-IDMA system

\begin{tabular}{|c|c|c|c|c|c|}
\hline Particulars & Channel & CFO & USERS & $\begin{array}{l}\text { SNR } \\
(\mathrm{dB})\end{array}$ & BER \\
\hline \multirow{2}{*}{ 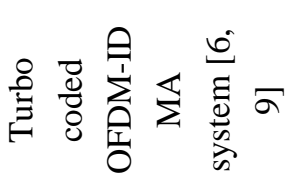 } & AWGN & 0.1 & 4 & 8 & $1 * 10^{-4}$ \\
\hline & Rayleigh & 0.1 & 4 & 15 & $7 * 10^{-3}$ \\
\hline \multirow{2}{*}{ 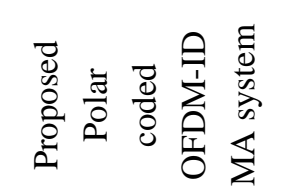 } & AWGN & 0.1 & 10 & 8 & $7.316^{*} 10^{-5}$ \\
\hline & Rayleigh & 0.1 & 10 & 15 & $1.021 * 10^{-4}$ \\
\hline
\end{tabular}

\section{CONCLUSIONS}

In this paper polar coded OFDM-IDMA system is implemented on raspberry pi in existence of slow fading multipath Rayleigh and AWGN channel. Further, 64 QAM modulation scheme is considered to assess BER against the effect ofparameters such as number of users and CFO for 2048 subcarriers. It is observed that the proposed framework within the sight of Rayleigh channel with CFO of 0.1 and
SNR of $15 \mathrm{dBfor} 10$ users improves the BER to $1.021 * 10-4$ However, the current Turbo coded OFDM-IDMA framework with CFO of 0.1 and SNR of $15 \mathrm{~dB}$ for fourusers accomplishes BER of just 7*10-3with QPSK modulation scheme. Thus enhancement of BER and four times network capacity is achieved using proposed system. Further, in the presence of AWGN channel withCFO of 0.1 at SNR of 8 $\mathrm{dB}$ for 10 number of users, 
polar coded OFDM-IDMA systemachieves BERof 7.316*10-5. It is superior as compared to turbo coded OFDM-IDMA system accomplishing BER of $1 * 10$-4for four users.

\section{REFERENCES}

[1] Nee, R. V., \& Prasad, R. (2000). OFDM for wireless multimedia communications. ArtechHouse,Inc.

[2] Proakis, J. G., \&Masaud, S. (2014). Digital Communication. Fifth Edition. Mc Graw Hill Education.

[3] Lathi, B. P., \& Ding, Z. (2010). Modern Digital and Analog Communication Systems. Fourth Edition. Oxford University Press, Inc.

[4] Trivedi, V. K., Ramadan, K., Kumar, P., Dessouky, M. I., \& El-Samie, F. E. A. (2019). Enhanced OFDM-NOMA for next generation wireless communication: A study of PAPR reduction and sensitivity to $\mathrm{CFO}$ and estimation errors. AEU-International Journal of Electronics and Communications, 102, 9-24.

[5] Singh, D., \& Joshi, H. D. (2019). Error probability analysis of STBC-OFDM systems with CFO and imperfect CSI over generalized fading channels. AEU-International Journal of Electronics and Communications, 98, 156-163.

[6] Wang, T., \&Liew, S. C. (2016). Frequency-Asynchronous Multiuser Joint Channel-Parameter Estimation, CFO Compensation, and Channel Decoding. IEEE Transactions on Vehicular Technology, 65(12), 9732-9746.

[7] Balogun, M. B., Oyerinde, O. O., \&Mneney, S. H. (2014). Adaptive Correction Algorithm for OFDM-IDMA Systems with Carrier Frequency OFFSET in a Fast Fading Multipath Channel. Journal of Cyber Security and Mobility, 2(4), 201-220.

[8] Balogun, M. B., Oyerinde, O. O., \&Mneney, S. H. (2013). Adaptive Correction Algorithm for OFDM-IDMA Systems with Carrier Frequency OFFSET in a Fast Fading Multipath Channel. Journal of Cyber Security and Mobility, 2(4), 201-220.

[9] Peng, T., Xiao, Y., He, X., \& Li, S. (2012). Improved detection of uplink OFDM-IDMA signals with carrier frequency offsets. IEEE Communications Letters, 16(5), 646-649.

[10] Xie, B., Qiu, W., \&Minn, H. (2012). Exact signal model and new carrier frequency offset compensation scheme for OFDM. IEEE Transactions on Wireless Communications, 11(2), 550-555.

[11] Oyerinde, O. O. (2018). Regularized Recursive Least Square-Based Time Domain Iterative Channel Estimation Scheme for OFDM-IDMA Systems. Circuits, Systems, and Signal Processing, 37(2), 831-846.

[12] Taşpınar, N., \&Şimşir, Ş. (2018). Pilot tones design using particle swarm optimization for OFDM-IDMA system. Neural Computing and Applications, 1-10.

[13] Yadav, M., Shokeen, V., \&Singhal, P. K. (2017). Flip left-right approach based novel inverse tree interleavers for IDMA scheme. AEU-International Journal of Electronics and Communications, 81, 182-191.

[14] Belaoura, W., Ghanem, K., \&Djeddou, M. (2015, July). Performance of OFDM-IDMA and OFDM-CDMA architectures over frequency selective MISO channels. In Antennas and Propagation \& USNC/URSI National Radio Science Meeting, 2015 IEEE International Symposium on (pp. 139-140). IEEE.

[15] Deng, X., Sha, J., Zhou, X., You, X., \& Zhang, C. (2017, October). Joint detection and decoding for polar-coded OFDM-IDMA systems. In 2017 IEEE 12th International Conference on ASIC (ASICON) (pp. 796-799). IEEE.

[16] Elkelesh, A., Ebada, M., Cammerer, S., \& ten Brink, S. (2018). Belief propagation list decoding of polar codes. IEEE Communications Letters, $22(8), 1536-1539$

[17] Abbas, S. M., Fan, Y., Chen, J., \&Tsui, C. Y. (2017). High-throughput and energy-efficient belief propagation polar code decoder. IEEE Transactions on Very Large Scale Integration (VLSI) Systems, 25(3), 1098-1111.

[18] Arikan, E. (2009). Channel polarization: A method for constructing capacity-achieving codes for symmetric binary-input memoryless channels. IEEE Transactions on Information Theory, 55(7), 3051-3073.

[19] Matary, D. E. E., Hagras, E. A., \& Abdel-Kader, H. M. (2018) Performance of Polar Codes for OFDM-Based UWB Channel. Journal of Computer and Communications, 6(03), 102.

[20] Arıkan, E., Costello, D. J., Kliewer, J., Lentmaier, M., Siegel, P., Urbanke, R., \&Pursley, M. (2016). Guest Editorial Recent Advances in Capacity Approaching Codes. IEEE Journal on Selected Areas in Communications, 34(2), 205-208.

[21] Vangala, H., Viterbo, E., Hong, Y. (2015, January). A Comparative Study of Polar Code Constructions for the AWGN Channel.
[22] Hui, D., Sandberg, S., Blankenship, Y., Andersson, M., \&Grosjean, L. (2018). Channel Coding in 5G New Radio: A Tutorial Overview and Performance Comparison with $4 \mathrm{G}$ LTE. ieee vehicular technology magazine, 13(4), 60-69.

[23] Cho, Y., Yonsei, J., Yang, W., \& Kang, C. (2010). MIMO-OFDM Wireless Communications with MATLAB. John Wiley \& Sons (Asia) Pte. Ltd. 